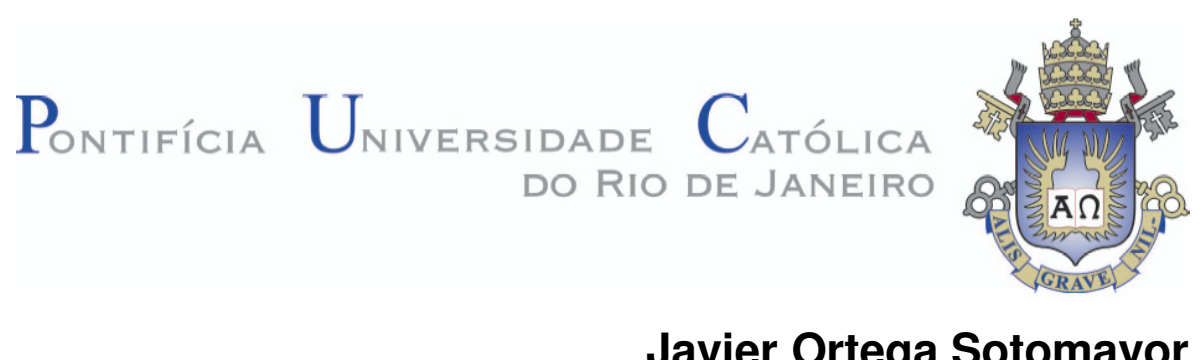

\title{
Avaliação da Coerência entre Dispositivos de Controle no Problema de Fluxo de Potência
}

Dissertação de Mestrado

Dissertação apresentada ao Programa de Pósgraduação em Engenharia Elétrica da PUC-Rio como requisito parcial para obtenção do grau de Mestre em Engenharia Elétrica.

Orientador : Prof. Ricardo Bernardo Prada Co-Orientador: Prof. João Alberto Passos Filho 


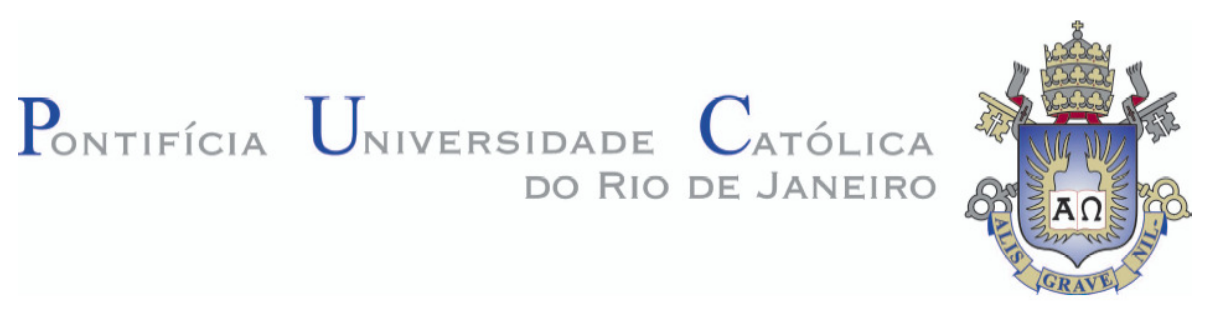

Javier Ortega Sotomayor

\title{
Avaliação da Coerência entre Dispositivos de Controle no Problema de Fluxo de Potência
}

\begin{abstract}
Dissertação apresentada como requisito parcial para obtenção do grau de Mestre pelo Programa de Pós-Graduação em Engenharia Elétrica do Departamento de Engenharia Elétrica do Centro Técnico Científico da PUC-Rio. Aprovada pela Comissão Examinadora abaixo assinada.
\end{abstract}

\author{
Prof. Ricardo Bernardo Prada \\ Orientador \\ Departamento de Engenharia Elétrica -- PUC-Rio \\ Prof. João A. Passo Filho \\ Co-Orientador \\ Departamento de Engenharia Elétrica -- UFJF
}

Prof. Ricardo Mota Henriques CEPEL

Prof. Tatiana Mariano Lessa de Assis UFRJ

Prof. José Eugenio Legal Coordenador Setorial do Centro

Técnico Cientifico-PUC-Rio

Rio de Janeiro, 29 de março de 2012. 
Todos os direitos reservados. É proibida a reprodução total ou parcial do trabalho sem autorização da autora, do orientador e da universidade.

\section{Javier Ortega Sotomayor}

Graduou-se em Engenharia Elétrica pela Universidade Nacional San Antonio Abad do Cusco - UNSAAC-Cusco (Cusco, Perú) em 2009.

Ficha Catalográfica

Ortega Sotomayor, Javier

Avaliação da coerência entre dispositivos de controle no problema de fluxo de potência / Javier Ortega Sotomayor, ; orientador: Ricardo Bernardo Prada ; co-orientador: João Alberto Passos Filho. - 2012.

144 f. : il. (color.) ; $30 \mathrm{~cm}$

Dissertação (mestrado)-Pontifícia Universidade Católica do Rio de Janeiro, Departamento de Engenharia Elétrica, 2012.

Inclui bibliografia

1. Engenharia elétrica - Teses. 2. Controle de tensão. 3. Áreas de controle de tensão. 4. Equipamentos controladores de tensão. 5. Autovalores e autovetores. I. Prada, Ricardo Bernardo. II. Passos Filho, João Alberto. III. Pontifícia Universidade Católica do Rio de Janeiro. Departamento de Engenharia Elétrica. IV. Título.

CDD: 621.3 
Dedico este trabalho à memória de meu querido papai:

Abel 


\section{Agradecimentos}

A Deus, por tudo.

A minha amada família, minha mamãe Lucia, minha tia Nélida, meu irmão Paul, pelo amor, confiança, bons conselhos e apoio constante.

Agradeço especialmente, a meu orientador Ricardo Bernardo Prada pela orientação nos diferentes aspectos relacionados ao tema de pesquisa.

Meu profundo agradecimento ao meu orientador João Alberto Passos Filho pela confiança, permanente apoio, orientação e paciência nas diferentes etapas do desenvolvimento desta dissertação.

Ao CNPq e à PUC-Rio, pelos auxílios financeiros concedidos, sem os quais este trabalho não poderia ter sido realizado.

Aos professores do departamento de Engenharia Elétrica; pelos ensinamentos.

Aos membros da comissão de Pós-graduação do DEE, pela oportunidade proporcionada de estudar meu mestrado na PUC-Rio, no Brasil.

A todos meus amigos do Laboratório de Sistemas de Energia, muito especialmente a Jelitza Ceballos, Erika Telles, Luz Angela Molina, Angela Padilla, Omar Galvéz, Jose Luis Choque, Juan Carlos Vargas e Oscar Cuaresma pelo apoio no desenvolvimento desta dissertação.

A meus amigos Luciana, Tatiana, Lorena, Américo, Juan Carlos, Amilcar, Ronald, Elder, Marco, Giancarlo, Martin, Presvitero, Emerson, Ricardo, Ernesto, Ivan e Wilfredo, pela companhia e amizade ao longo destes anos no Brasil. 


\section{Resumo}

Sotomayor, Javier Ortega; Prada, Ricardo B. Avaliação da Coerência entre Dispositivos de Controle no Problema de Fluxo de Potência. Rio de Janeiro, 2012. 144p. Dissertação de Mestrado - Departamento de Engenharia Elétrica, Pontifícia Universidade Católica do Rio de Janeiro.

Com o aumento do número de dispositivos de controle representados nos casos práticos, pode ser verificado o aparecimento de interações entre suas ações de controle. Quando estas interações não são coordenadas podem ocasionar a diminuição da eficiência do método de Newton-Raphson no problema de fluxo de potência, resultando em convergências lentas e frequentemente soluções oscilatórias ou até mesmo a divergência do método. Uma adequada identificação destas interações pode contribuir para tomar as medidas corretivas necessárias e assim evitar este tipo de problema. Com esse objetivo, identificam-se as interações entre múltiplos dispositivos de controle (mais de dois equipamentos de controle) a partir da análise dos autovalores e fatores de participação da matriz de sensibilidade de controles denominada [MSC]. Esta matriz, elaborada com base num modelo alternativo para a representação do controle de tensão local das barras PV, é obtida da redução da matriz Jacobiana expandida do problema de fluxo de potência. Dentro deste contexto, se verifica a presença de autovalores que apresentam informações similares sobre os dispositivos de controle com fortes interações entre suas ações de controle, desenvolvendo-se assim, um método baseado no conceito de colinearidade capaz de identificar e agrupar estes autovalores. Os resultados da avaliação do método desenvolvido aplicado em sistemas de pequeno e grande porte mostram a relevância e a viabilidade da utilização prática dos desenvolvimentos propostos neste trabalho.

\section{Palavras-chave}

Controle de tensão; áreas de controle de tensão; equipamentos controladores de tensões; autovalores e autovetores. 


\section{Abstract}

Sotomayor, Javier Ortega; Prada, Ricardo B. (Advisor). Interdependence of Voltage Control Equipments: Coherency Assessment in the Power Flow Poblem. Rio de Janeiro, 2012. 144p. MSc. Dissertation - Departamento de Engenharia Elétrica, Pontifícia Universidade Católica do Rio de Janeiro.

The increasing number of control devices represented in practical cases, we can see the appearance of interactions between their control actions. When these interactions are not coordinated (conflict), the efficiency of NewtonRaphson method decrease to the power flow problem, the convergence is slow and the solutions are oscillatory. A correct identification of these interactions can help to take corrective actions and thus avoid this problem. With this objective, the identification of interactions between control devices (more than 2 control equipment) is established from the modal analysis of the sensitivity matrix [MSC]. This sensitivity matrix [MSC] is developed in based to alternative model to represent the local voltage control of the PV buses. This [MSC] is obtained from the reduction of the Jacobean matrix expanded of power flow problem. Within this context, it also checks for the presence of eigenvalues that have similar information about the significant interactions between control devices, thus developing a method based on the use index of sensitivity matrix [MSC] and concept of collinearity able to identify and group these eigenvalues. The results of the evaluation method applied to systems designed for small and large show the relevance and feasibility of practical use of proposed developments in this work.

\section{Keywords}

Voltage control; voltage control areas; voltage controller equipments; eigenvalues and eigenvectors. 


\section{Sumário}

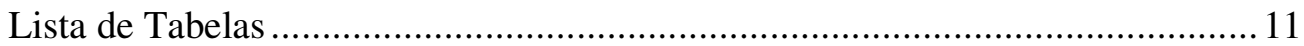

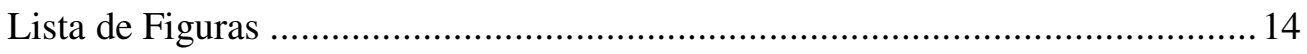

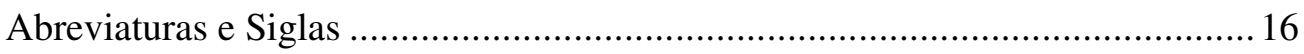

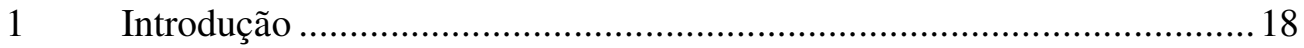

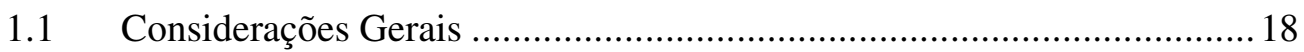

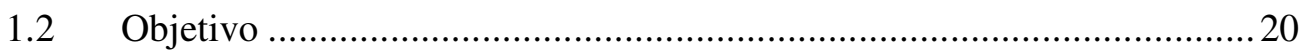

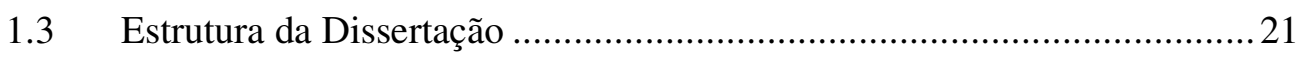

2 Matriz de Sensibilidade de Controles ..................................................22

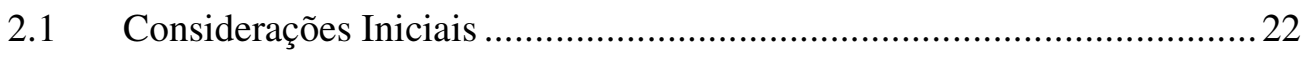

2.2 Representação Convencional do Controle Local de Tensão

das barras PV no Problema de Fluxo de Potência [3] .........................................22

2.3 Representação do Controle Local e Remoto de Tensão das

barras PV no Problema de Fluxo de Potência [7]...............................................2 23

2.3.1 Controle Local de Tensão das Barras PV (CLT) …...............................223

2.3.2 Controle Remoto de Tensão das Barras PV (CRT) ................................2 27

2.4 Modelo Utilizado para a Representação das Barras PV

no Problema de Fluxo de Potência [6] .............................................................2 29

2.5 Matriz de Sensibilidade de Controles incluindo o Modelo

utilizado para a Representação das Barras PV no problema de FP ...................... 32

2.5.1 Sistema de Equações Linearizadas ........................................................ 32

2.5.2 Aplicação em Sistema-Teste .............................................................. 34

2.6 Identificação de Interações entre Dispositivos de Controle com base

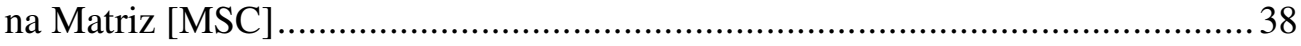

2.6.1 Autopropiedades: Autovalores e Autovetores ....................................... 38

2.6.2 Método de Identificação de Interações entre Dispositivos de Controle .... 41 
2.6.3 Algoritmo para a Identificação de interações entre Dispositivos

de Controle

2.7 Resultados do Método de Identificação de Interações entre

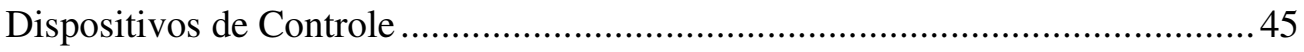

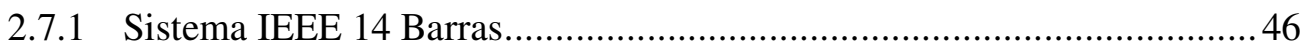

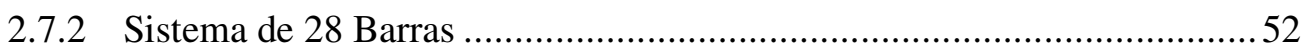

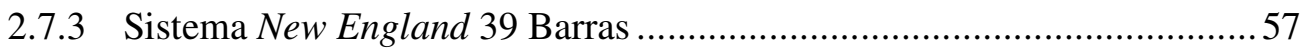

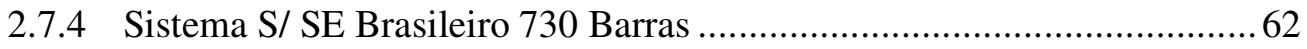

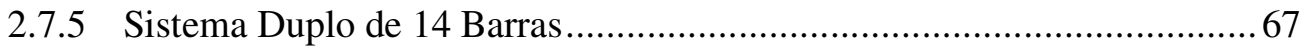

3 Identificação e Agrupamento dos Autovalores que possuem

Informaões Similares sobre as Interações dos Dispositivos de Controle ............. 80

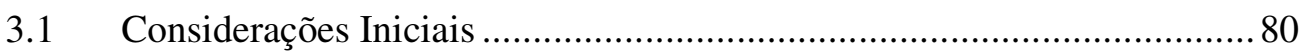

3.2 Método de Identificação e Agrupamento dos Autovalores por Índices

de Sensibilidade e Conceito de Colinearidade .................................................... 81

3.3 Algoritmo de Identificação e Agrupamento dos Autovalores .................. 89

3.4 Resultado do Método de Identificação e Agrupamento de Autovalores ... 92

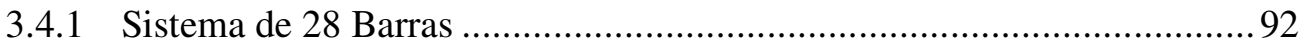

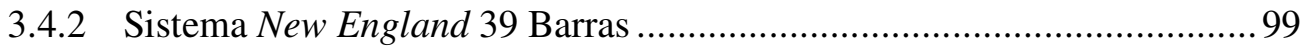

3.4.3 Sistema S/SE Brasileiro 730 Barras …............................................... 108

3.4.4 Sistema Duplo de 14 Barras............................................................... 112

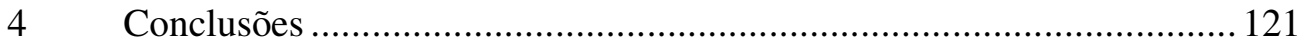

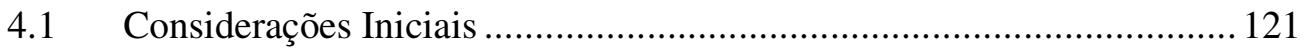

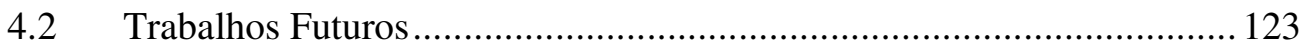

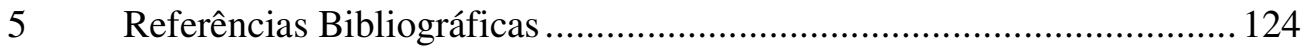

Apêndice A - Revisão do Método de Newton-Raphson para Solução do

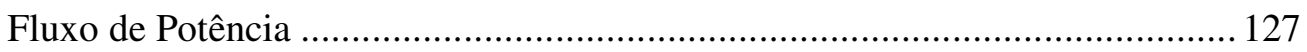

A.1 Considerações Iniciais ................................................................. 127

A.2 Método de Newton-Rapshon ...................................................... 128 
Apêndice B - Revisão do Método de Identificação e Análise da Interação

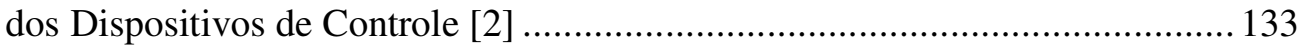

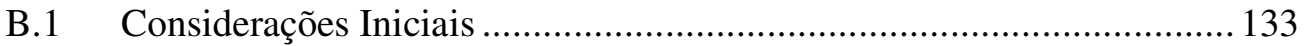

B.2 Descrição do Método......................................................................... 133

B.2.1 Análise Modal da Matriz de Sensibilidade de Controles.................... 135

Apêndice C - Arquivos de Dados de Entrada para o Programa Desenvolvido

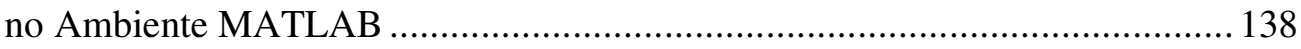

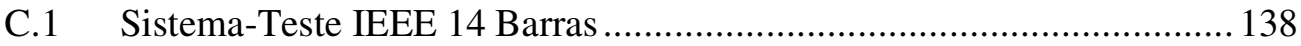

C.2 Sistema-Teste 28 Barras ...................................................................... 139

C.3 Sistema-Teste New England 39 Barras................................................. 141

C.4 Sistema Duplo 14 Barras .................................................................... 144 


\section{Lista de Tabelas}

Tabela 2.1 - Conjunto de dados e incógnitas do Diagrama Unifilar do

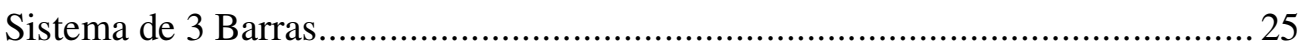

Tabela 2.2 - Ponto de Operação do Sistema-Teste de 5 Barras............................ 35

Tabela 2.3 - $\Delta$ X Após do Aumento de 0,01 pu na Barra 3................................ 37

Tabela 2.4 - Ponto de Operação do Sistema IEEE 14 Barras ............................. 46

Tabela 2.5 - Autovalores da Matriz [MSC] do Sistema 14 Barras ...................... 48

Tabela 2.6 - Resultados do Menor Autovalor $\left(\boldsymbol{\lambda}_{1}=0,020\right)$ da Matriz [MSC]...... 48

Tabela 2.7 - Resultados do Menor Autovalor $\left(\lambda_{1}=0,02\right)$ da Matriz [M]

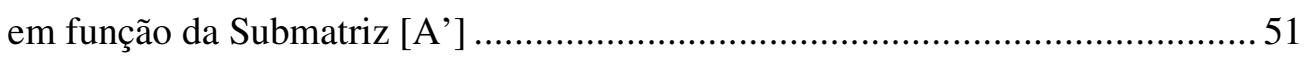

Tabela 2.8 - Características Principais do Sistema 28 Barras ........................... 52

Tabela 2.9 - Acoplamentos entre os Dispositivos de Controle nas

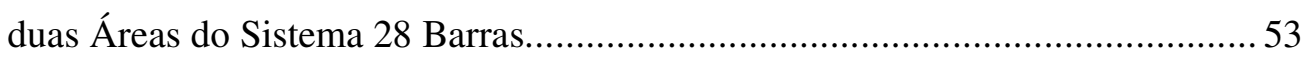

Tabela 2.10 - Autovalores da Matriz [MSC] do Sistema 28 Barras ................... 54

Tabela 2.11 - Fatores de Participação para os Autovalores selecionados ............. 55

Tabela 2.12 - Características Principais do Sistema New England 39................ 57

Tabela 2.13 - Acoplamentos entre os Dispositivos de controle em

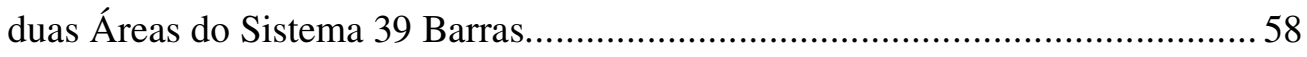

Tabela 2.14 - Autovalores da Matriz [MSC] do Sistema 39 Barras .................... 58

Tabela 2.15 - Fatores de Participação para os Autovalores $\lambda_{4}$ até $\lambda_{8} \ldots \ldots \ldots \ldots . . . . . . . .61$

Tabela 2.16 - Características Principais do Sistema S/SE Brasileiro

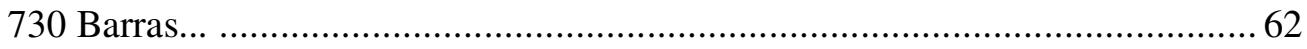

Tabela 2.17- Autovalores da Matriz [MSC] do Sistema 730 Barras ................... 64

Tabela 2.18 - Acoplamentos entre os Dispositivos de Controle em duas Áreas do Sistema Duplo de 14 Barras - Etapa 1......................................... 70

Tabela 2.19 - Ponto de Operação do Sistema Duplo de 14 Barras ...................... 70

Tabela 2.20 - Autovalores da Matriz [MSC] do Sistema Duplo 14 Barras

- Etapa 1

Tabela 2.21 - Avaliação da Convergência do Sistema Duplo de 14 Barras

-Etapa 1 
Tabela 2.22 - Potência Reativa dos Geradores analisados - Etapa 1

Tabela 2.23 - Acoplamentos entre os Dispositivos de Controle

nas duas nas duas Áreas Sistema Duplo de 14 Barras - Etapa 2 ........................ 74

Tabela 2.24 - Autovalores da Matriz [MSC] do Sistema Duplo 14 Barras

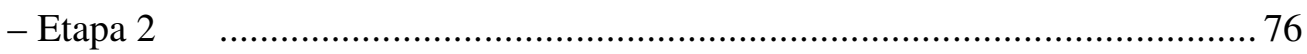

Tabela 2.25 - Avaliação da Convergência do Sistema Duplo de 14 Barras

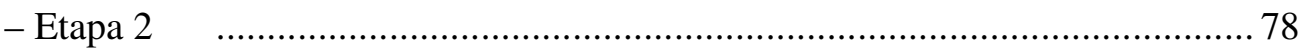

Tabela 2.26 - Potência Reativa dos Geradores e tapes dos LTCs

analisados - Etapa 2

Tabela 3.1 - Autovalores da Matriz [MSC] do Sistema Duplo 14 Barras

- Etapa 1

Tabela 3.2 - Faixas e Cores para os Valores de IC .......................................... 87

Tabela 3.3 - Menores Autovalores da Matriz [MSC] do Sistema 28 Barras........ 93

Tabela 3.4 - Fatores de Participação para os Autovalores Selecionados ............. 93

Tabela 3.5 - Índices de Sensibilidade dos Dispositivos de Controle

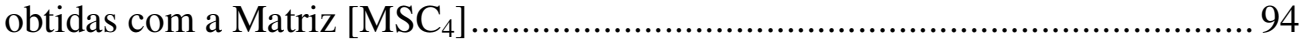

Tabela 3.6 - Índice de Sensibilidade dos Dispositivos de Controle calculadas

a partir das Matrizes $\left[\mathrm{MSC}_{4}\right]_{\lambda \mathrm{i}}$ obtidas pela contribuiç̧ão de cada Autovalor ....... 95

Tabela 3.7 - Índices de Colinearidade entre as Sensibilidades

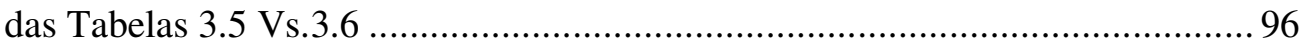

Tabela 3.8 - Índice de Sensibilidade obtidas pela aplicação do Critério a).......... 98

Tabela 3.9 - Características Principais do Sistema New England 39 Barras ..... 100

Tabela 3.10 - Menores Autovalores da Matriz [MSC] do Sistema 39 Barras.... 101

Tabela 3.11 - Fatores de Participação para os Autovalores Selecionados ......... 101

Tabela 3.12 - Índices de Sensibilidade dos Dispositivos de Controle

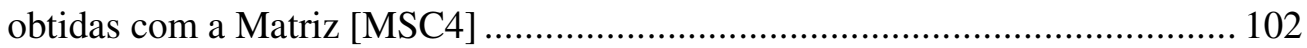

Tabela 3.13 - Índice de Sensibilidade dos Dispositivos de Controle

calculadas a partir das Matrizes $\left[\mathrm{MSC}_{4}\right]_{\lambda_{\mathrm{i}}}$ obtidas pela contribuição de cada

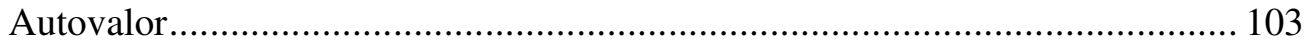

Tabela 3.14 - Índices de Colinearidade entre as Sensibilidades

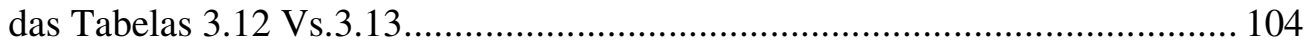

Tabela 3.15 - Índice de Sensibilidades obtidas pela aplicação do Critério a) .... 107

Tabela 3.16 - Características Principais do Sistema S/SE Brasileiro 
730 Barras

Tabela 3.17 - Índices de Colinearidade entre as Sensibilidades

de $\mathrm{X}_{\lambda_{\mathrm{i}}}$ vs. $\mathrm{Y}_{\lambda_{\mathrm{i}}}$ 110

Tabela 3.18 - Menores Autovalores da Matriz [MSC] do Sistema de

Duplo 14 Barras - Etapa 2

Tabela 3.19 - Fatores de Participação para os Autovalores Selecionados

Tabela 3.20 - Índices de Sensibilidade dos Dispositivos de Controle obtidas com a Matriz $\left[\mathrm{MSC}_{4}\right]$

Tabela 3.21 - Índice de Sensibilidade dos Dispositivos de Controle

calculadas a partir das Matrizes $\left[\mathrm{MSC}_{4}\right]_{\lambda_{\mathrm{i}}}$ obtidas pela contribuição de cada

Autovalor

Tabela 3.22 - Índices de Colinearidade entre as Sensibilidades das Tabelas

3.20 Vs. 3.21

Tabela 3.23 - Índice de Sensibilidade dos Dispositivos de Controle utilizando

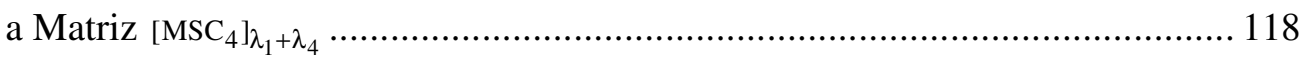

Tabela 3.24 - Índice de Sensibilidade dos Dispositivos de controle utilizando a matriz $\left[\mathrm{MSC}_{4}\right] \lambda_{2}+\lambda_{3}$

Tabela 3.25 - Índices de Colinearidade entre as Sensibilidades das Tabelas 3.20 Vs. 3.23

Tabela 3.26 - Índices de Colinearidade entre as Sensibilidades das Tabelas

3.20 Vs.3.24 


\section{Lista de Figuras}

Figura 2.1 - Diagrama Unifilar do Sistema de 3 barras e 3 circuitos................... 25

Figura 2.2 - Diagrama Unifilar do Sistema-Teste de 5 Barras ........................... 35

Figura 2.3 - Fluxograma do Algoritmo para a Identificação de Interações entre

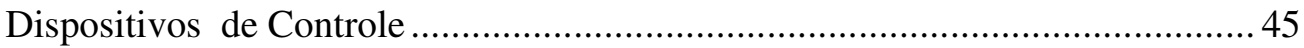

Figura 2.4 - Diagrama Unifilar do Sistema IEEE 14 Barras .............................. 46

Figura 2.5 - Potência Reativa gerada pelas Máquinas 1, 2 e 3 em função à $V_{2} \ldots 51$

Figura 2.6 - Diagrama Unifilar do Sistema 28 Barras....................................... 52

Figura 2.7 - Mode-Shape de $\lambda_{1}$ de [MSC] do Sistema de 28 Barras.................... 55

Figura 2.8 - Mode-Shape de $\lambda_{3}$ de [MSC] do Sistema de 28 Barras....................56

Figura 2.9 - Diagrama Unifilar do Sistema New England 39 Barras.................. 57

Figura 2.10 - Fatores de Participação de $\lambda_{1}, \lambda_{2}$ e $\lambda_{3}$ de [MSC] do Sistema New

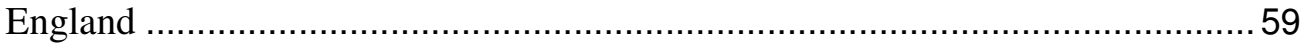

Figura 2.11 - Mode-Shape de $\lambda_{1}$ de [MSC] do Sistema de New England............ 60

Figura 2.12 - Mode-Shape de $\lambda_{2}$ de [MSC] do Sistema de New England............ 60

Figura 2.13 - Mode-Shape de $\lambda_{3}$ de [MSC] do Sistema de New England............ 60

Figura 2.14 - Topologia da Região do Área 1 que indica os Acoplamentos dos

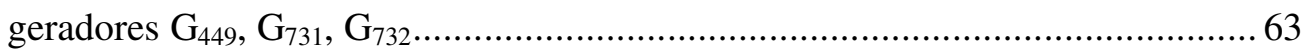

Figura 2.15 - Topologia da Região do Área 5 que indica os Acoplamentos dos

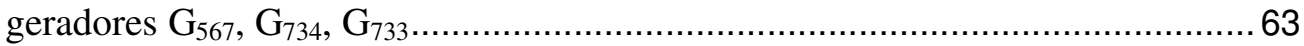

Figura 2.16 - Fatores de Participação de $\lambda_{1}$ e $\lambda_{3}$ de [MSC] do Sistema

730 Barras 65

Figura 2.17 - Mode-Shape de $\lambda_{1}$ e $\lambda_{3}$ de [MSC] do Sistema 730 Barras 65

Figura 2.18 - Fatores de Participação de $\lambda_{2}$ e $\lambda_{4}$ de [MSC] do Sistema de 730

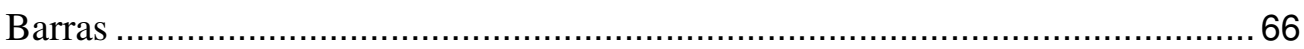

Figura 2.19 - Mode-Shape de $\lambda_{2}$ e $\lambda_{4}$ de [MSC] do Sistema 730 Barras .............. 66

Figura 2.20 - Diagrama Unifilar do Sistema Duplo de 14 Barras ....................... 67

Figura 2.21 - Diagrama Unifilar do Sistema duplo de 14 Barras - Etapa 1 .........6 68

Figura 2.22 - Diagrama Unifilar do Sistema Duplo de 14 Barras - Etapa 2 ....... 69

Figura 2.23 - Fatores de Participação de $\lambda_{1}$ e $\lambda_{2}$ de [MSC] do Sistema Duplo 
14 Barras - Etapa 1

Figura 2.24 - Mode-Shape de $\lambda_{1}$ e $\lambda_{2}$ de [MSC] do Sistema Duplo 14 Barras -

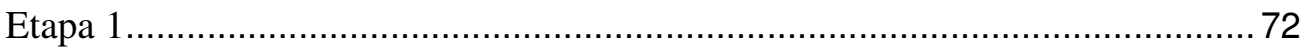

Figura 2.25 - Fatores de Participação de $\lambda_{1}$ e $\lambda_{4}$ de [MSC] do Sistema Duplo

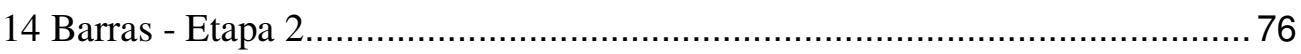

Figura 2.26 - Mode-Shape de $\lambda_{1}$ e $\lambda_{4}$ de [MSC] do Sistema Duplo 14 Barras -

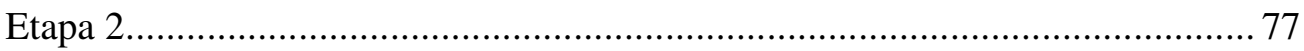

Figura 2.27 - Fatores de Participação de $\lambda_{2}$ e $\lambda_{3}$ de [MSC] do Sistema Duplo

14 barras - Etapa 2

Figura 2.28 - Mode-Shape de $\lambda_{2}$ e $\lambda_{3}$ de [MSC] do Sistema Duplo 14 Barras -

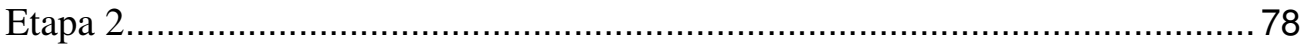

Figura 3.1 - Diagrama Unifilar do Sistema Duplo de 14 Barras - Etapa 1.......... 83

Figura 3.2 - Fluxograma do Algoritmo de Identificação e Agrupamento de

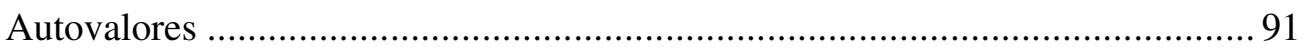

Figura 3.3 - Diagrama Unifilar do Sistema 28 Barras..................................... 93

Figura 3.4 - Diagrama Unifilar do Sistema New England 39 Barras................. 100

Figura 3.5 - Diagrama Unifilar Parcial do S/SE Brasileiro 730 de Barras......... 109

Figura 3.6 - Diagrama Unifilar do Sistema Duplo de 14 Barras ...................... 112 


\section{Abreviaturas e Siglas}

\begin{tabular}{|c|c|}
\hline ANEEL & Agencia Nacional de Energia Elétrica \\
\hline CEPEL & Centro de Pesquisas de Energia Elétrica \\
\hline ONS & Operador Nacional do Sistema \\
\hline IEEE & Institute of Electrical and Electronics Engineers \\
\hline SEP & Sistema Elétrico de Potência \\
\hline SEPs & Sistemas Elétricos de Potência \\
\hline LTC & Load Tap Changer \\
\hline CER & Compensador Estático de Reativos \\
\hline HVDC & Corrente Contínua em Alta Tensão \\
\hline CLT & Controle Local de tensão \\
\hline CRT & Controle Remoto de Tensão \\
\hline AS & Ajuste Simultâneo \\
\hline$F P$ & Fator de Participação \\
\hline $\mathrm{P}$ & Potência Ativa \\
\hline Q & Potência Reativa \\
\hline $\mathrm{S} / \mathrm{SE}$ & Sul / Sudeste \\
\hline$\theta$ & Ângulo da Tensão \\
\hline $\mathrm{V}$ & Módulo da Tensão \\
\hline$\Delta \mathrm{P}$ & Variação incremental de potência ativa \\
\hline$\Delta \mathrm{Q}$ & Variação incremental de potência reativa \\
\hline$\Delta \theta$ & Variação incremental do ângulo da tensão \\
\hline$\Delta \mathrm{V}$ & Variação incremental do módulo da tensão \\
\hline$\Delta \mathrm{Q}_{\mathrm{G}}$ & Variação incremental de potência reativa do gerador \\
\hline$[\mathrm{J}]$ & Matriz Jacobiana \\
\hline$[\mathrm{A}]$ & Submatriz da matriz Jacobiana expandida \\
\hline [B] & Submatriz da matriz Jacobiana expandida \\
\hline$[\mathrm{C}]$ & Submatriz da matriz Jacobiana expandida \\
\hline [D] & Submatriz da matriz Jacobiana expandida \\
\hline$\partial \mathrm{p} / \partial \theta$ & Derivada parcial da potência ativa em relação ao ângulo da tensão \\
\hline$\partial \mathrm{P} / \partial \mathrm{V}$ & Derivada parcial da potência ativa em relação ao módulo da tensão \\
\hline
\end{tabular}


$\partial \mathrm{P} / \partial \mathrm{t} \quad$ Derivada parcial da potência ativa em relação ao tape de um LTC

$\partial \mathrm{P} / \partial \mathrm{Q}_{\mathrm{G}} \quad$ Derivada parcial da potência ativa em relação à potência reativa gerada por um gerador ou compensador síncrono

$\partial \mathrm{Q} / \partial \theta$ Derivada parcial da potência reativa em relação ao ângulo da tensão

$\partial \mathrm{Q} / \partial \mathrm{V}$ Derivada parcial da potência reativa em relação ao módulo da tensão

$\partial \mathrm{Q} / \partial \mathrm{t} \quad$ Derivada parcial da potência reativa em relação ao tape de um LTC

$\partial \mathrm{Q} / \partial \mathbf{Q}_{\mathrm{G}} \quad$ Derivada parcial da potência reativa em relação à potência reativa gerada por um gerador ou compensador síncrono

IC Índice de Colinearidade

IS Índice de Sensibilidade

$\lambda \quad$ Autovalor

$\varphi \quad$ Autovetor à direita

$\psi \quad$ Autovetor à esquerda

[MSC] Matriz de sensibilidade de controles

[MSC $\left.{ }_{1}\right]$ Submatriz 1 da matriz de sensibilidade de controles inversa

[MSC $\mathrm{MS}_{2}$ Submatriz 2 da matriz de sensibilidade de controles inversa

[MSC $\mathrm{MS}_{3}$ Submatriz 3 da matriz de sensibilidade de controles inversa

[ $\left.\mathrm{MSC}_{4}\right]$ Submatriz 4 da matriz de sensibilidade de controles inversa 